\title{
Effective Analysis of Dicyclanil in Lamb and Chicken Muscle using HPLC- $\mathrm{UV} / \mathrm{Vis}$ and LC/MS/MS
}

\author{
ByungJu Kim and Seung-Woon Myung* \\ Department of Chemistry, Kyonggi University, Suwon, 443-760, Republic of Korea
}

Received May 18, 2011; Revised June 13, 2011; Accepted June 13, 2011

First published on the web June 15, 2011; DOI: 10.5478/MSL.2011.2.2.045

\begin{abstract}
The authors describe a method for monitoring dicyclanil levels in lamb and chicken muscle tissues. The devised procedure involves dicyclanyl extraction by SPE and its detection HPLC-UV/Vis and LC/MS/MS. The method was found to have LOD and LOQ values of $0.02 \mathrm{mg} \mathrm{kg}^{-1}$ and $0.05 \sim 0.06 \mathrm{mg} \mathrm{kg}^{-1}$, respectively. The intraday precision and an accuracy of spiked samples were found to have $2.3 \sim 10.4 \mathrm{RSD} \%$ and $80.9 \sim 105.7 \%$, respectively.
\end{abstract}

Key words: Dicyclanil, Lamb, Chicken, Veterinary Drugs, HPLC, LC/MS/MS

\section{Introduction}

Veterinary drugs are widely used to treat and prevent disease, and are administered to livestock in feed or drinking water. $^{1-2}$ However, the improper use of these drugs can leave residues in tissues, which can lead to health problems for human who consumes the animal products. Dicyclanil (Figure 1) is a pyrimidine-derived insect growth regulator that is used by veterinarians to prevent myiasis or fly-strike, ${ }^{3-4}$ but the presence of residual dicyclanil, has received much attention because it has become a consumer safety issue. The Korean Food and Drug Administration had established a maximum residue limit (MRL) of $0.15 \mathrm{mg} / \mathrm{kg}$ of dicyclanil in sheep and chicken muscle.

The development of simple and rapid methods for the determination of dicyclanil levels in sheep and chicken muscle meat has become increasing important. Few methods have<smiles>N#Cc1c(N)nc(NC2CC2)nc1Nc1ccccc1</smiles>

Figure 1. Chemical structure of dicyclanil.

*Reprint requests to Dr. Seung-Woon Myung

E-mail: swmyung@kgu.ac.kr been devised for dicyclanil determination, ${ }^{5-7}$ and those that have been described are based on liquid-liquid extraction. These methods are time-consuming and lack precision.

Here, the authors describe an effective and sensitive preparation method for dicyclanil in sheep and chicken muscle based on SPE (solid phase extraction) followed by HPLC-UV/Vis and LC/MS/MS.

\section{Experimental}

\section{Reagents, Materials, and Instruments}

Dicyclanil standard solution was prepared using high purity reagents purchased from Sigma-Aldrich (St Louis, MO). Organic solvents, such as methanol, acetonitrile, chloroform, and acetone, were obtained from J. T. Baker (NJ, USA). Heptafluobutyric acid (HFBA) was obtained from SigmaAldrich (St Louis, MO, USA).

The supra $22 \mathrm{~K}$ centrifuge was purchased from Han-Il Scientific Co. Ltd. (Seoul), GF/B glass filter paper from Whatman (Maidstone, UK), and syringe disc filters $(0.45 \mu \mathrm{m}$ and $13 \mathrm{~mm})$ from Life Sciences (UK). The Oasis HLB (HydrophilicLipophilic Balance, $200 \mathrm{mg}, 6 \mathrm{cc}$ ) SPE cartridge used for analyte extraction was purchased from Waters (Milford, Massachusetts), and the concentrator was a TurboVap LV nitrogen unit from Caliper Lifescience (Seattle, WA).

An Agilent 1100 series HPLC system (Palo Alto, CA) equipped with a binary pump was connected to an Agilent G1313A autosampler and G1313A UV-Vis detector. The analytical column used was a reverse-phase CAPCELL PAK C18 column $(250 \mathrm{~mm} \times 4.6 \mathrm{~mm}, 5 \mu \mathrm{m}$ particle size $)$. Mobile phase $\mathrm{A}$ (20 mM HFBA) and phase $\mathrm{B}$ (methanol:acetonitrile = $1: 1.8)$ were used with a flow rate of $1.0 \mathrm{~mL} / \mathrm{min}$. Separation was achieved using the following linear gradient system: $\mathrm{A}: \mathrm{B}=75: 25(\mathrm{v} / \mathrm{v}), 3 \min \mathrm{A}: \mathrm{B}=75: 25,16 \min \mathrm{A}: \mathrm{B}=65: 35$, $16.5 \min \mathrm{A}: \mathrm{B}=75: 25$, and $21.5 \mathrm{~min} \mathrm{~A}: \mathrm{B}=75: 25$. $\mathrm{A} 10 \mathrm{~min}$ 
delay was allowed post-injection for column re-equilibration. The injection volume was $20 \mu \mathrm{L}$. Analytes were detected with the DAD (Diode Array Dector) set at $262 \mathrm{~nm}$.

Confirmatory analysis was performed using an Agilent 1200 series HPLC (Agilent Technologies, Palo Alto, CA, USA) equipped with a sample auto injector (Agilent 1200 series Autosampler) and an Agilent 6410 Triple Quadrupole tandem mass spectrometer. Nitrogen was used as the desolvation gas (gas flow $10 \mathrm{~L} / \mathrm{min}$ at $350{ }^{\circ} \mathrm{C}$ ) and the capillary voltage was set at $3.5 \mathrm{kV}$ under the condition of ESI $(+)$ mode.

Analyses were carried out in MRM (multiple reaction monitoring) mode. The first step for analyzing the standards was to monitor the full scan of the mass spectrum under the scan mode. The precursor ion of each analyte is selected in order for perfect collision energy which leads to following product ions. And multiple reaction monitoring (MRM) was then performed by the selected characteristic ions.

\section{Sample preparation}

Samples $(10 \mathrm{~g})$ were homogenized using a mechanical homogenizer, treated with acetonitrile $(10 \mathrm{~mL})$, shaken for $30 \mathrm{~min}$ at $250 \mathrm{rpm}$, and centrifuged for $15 \mathrm{~min}$ at $3500 \mathrm{rpm}$. Collected supernatants were mixed with $1 \mathrm{~mL}$ of distilled water, evaporated to $1 \mathrm{~mL}$ using a nitrogen evaporator at
$60{ }^{\circ} \mathrm{C}$, and then $4 \mathrm{~mL}$ of distilled water was added. The samples $(5 \mathrm{ml})$ were then loaded into Oasis HLB (200 mg, $6 \mathrm{cc}$ ) cartridges conditioned with $8 \mathrm{ml}$ of methanol/DI water $(1: 1 \mathrm{vol} / \mathrm{vol})$, and eluted with $5 \mathrm{~mL}$ methanol (twice). Eluants were completely vaporized in a nitrogen evaporator at $60{ }^{\circ} \mathrm{C}$, and residues were dissolved in $200 \mu \mathrm{L}$ of solvent and then filtered through a $0.45 \mu \mathrm{m}$ disk filter for $\mathrm{LC} / \mathrm{UV}$-vis and LC/MS/MS analysis.

\section{Results and Discussion}

\section{Chromatographic separation and mass spectrometry}

It is difficult to retain dicyclanil in chromatographic reversed-phase mode, and therefore, a chromatographic mode based on the ion pair mode (heptafluorobutyric acid (HFBA)) was used. Dicyclanil was detected at $9.2 \mathrm{~min}$ using the reversed-phase liquid chromatographic system without any derivatization procedure.

LC/MS/MS analysis was used to confirm the detection of dicyclanil. The same HPLC conditions were used for LC/MS/MS and HPLC-UV/Vis. The mass spectrometer was operated in positive electrospray mode, and conditions were for multiple reaction monitoring (MRM). Full scan and collision induced dissociation (CID) tests were performed for MRM (Figure 2). The protonated molecular ion $\mathrm{m} / \mathrm{z} 191[\mathrm{M}+\mathrm{H}]^{+}$of dicyclanil
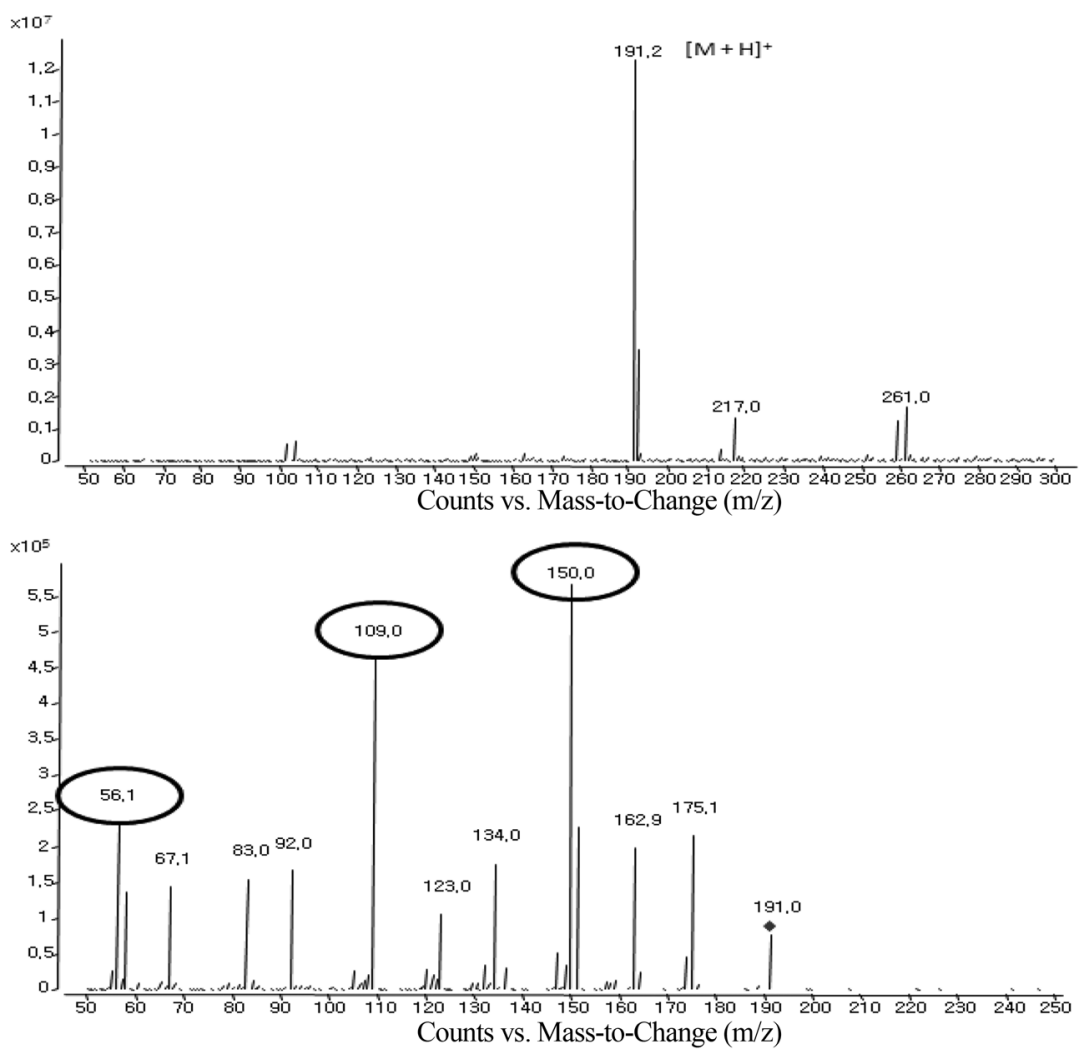

Figure 2. Full scan spectrum (upper) and product ion scan spectrum (lower) of dicyclanil (precursor ion:m/z 191): oval rings indicate the MRM ions. 
dominated the spectrum in positive ESI mode and it was selected as precursor ion. Characteristic fragment ions observed in product ion scan mode are presented in Figure 2. The criteria ion and the quantitation ion were obtained as the ions with $\mathrm{m} / \mathrm{z} 150$ and 109 , and $\mathrm{m} / \mathrm{z} 56$, respectively.

\section{Method validation}

Sample preparation was performed using an solid phase extraction technique. Limits of Detection (LOD) and Limits of Quantification (LOQ) for spiked blank muscle samples were determined by LC/UV-Vis.

The methods to determine LOD and LOQ values are described below. The LOD was assumed from the instrumental analysis of standard solution of dicyclanil, and samples $(n=7)$ were prepared at a concentration of 1-5 times the LOD.

After calculating standard deviations $(\sigma)$ and the slope (m) of the linear calibration curve using the results of 7 replicate, LOD and LOQ were found to be $3 \mathrm{\sigma} / \mathrm{m}$ and $10 \mathrm{\sigma} / \mathrm{m}$, respectively. As a result, the LOD and LOQ in lamb muscle were both between $0.02 \mathrm{mg} \mathrm{kg}^{-1}$ and $0.05 \mathrm{mg} \mathrm{kg}^{-1}$, respectively, and LOD and LOQ in chicken muscle were both between $0.02 \mathrm{mg} \mathrm{kg}^{-1}$ and $0.06 \mathrm{mg} \mathrm{kg}^{-1}$, respectively. These LOQs were above the MRL of the KFDA for chicken and lamb muscle.

To analyze residual analyte in samples, samples were spiked with standard solutions at a concentration of LOQ $\sim 6$ times of MRLs. Analytes were then extracted by the established analytical method. Calibration curves were obtained for the external standard method and the correlation coefficients for both drugs and matrices were reasonable $\left(\mathrm{r}^{2}=0.998\right.$; Figure 3$)$.

The accuracy and precision (expressed as RSD\%) in the concentration range $0.05 \sim 0.9 \mathrm{mg} \mathrm{kg}^{-1}$ in spiked lamb muscles were $96.1 \sim 112.4 \%$ and $1.0 \sim 5.4 \%$, respectively. The accuracy and precision for dicyclanil in the concentration range 0.06 $0.9 \mathrm{mg} \mathrm{kg}^{-1}$ in spiked chicken muscles were $80.9 \sim 102.5 \%$

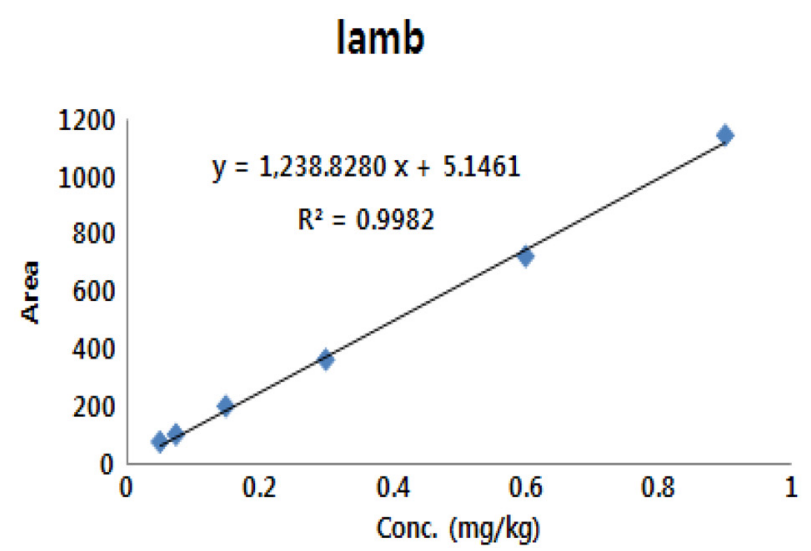

\section{Chicken}

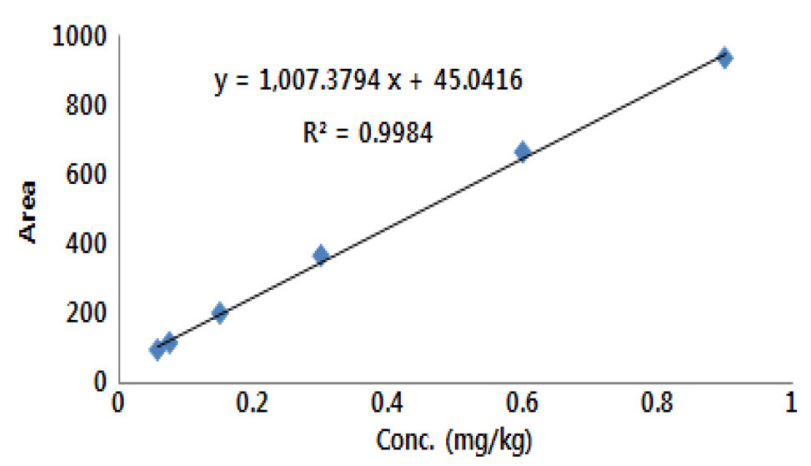

Figure 3. Calibration curves for the determination of dicyclanil in lamb and chicken muscle.

and $2.5 \sim 10.4 \%$, respectively. These results were well within the recommended acceptable values of $-30 \%$ to $+20 \%$ at each concentration (Table 1).

Table 1. LODs, LOQs, accuracy, and precision for the determination of dicyclanil in lamb and chicken muscle

\begin{tabular}{|c|c|c|c|c|c|c|}
\hline Com-pound & Matrices & LODs* (mg/kg) & LOQs** (mg/kg) & Conc. (mg/kg) & Accuracy*** (Recovery \%) & Precision (RSD \%) \\
\hline \multirow{12}{*}{ Dicyclanil } & \multirow{6}{*}{ Lamb } & \multirow{6}{*}{0.02} & \multirow{6}{*}{0.05} & 0.05 & 112.4 & 4.8 \\
\hline & & & & 0.075 & 104.3 & 5.1 \\
\hline & & & & 0.15 & 105.0 & 2.3 \\
\hline & & & & 0.3 & 96.1 & 5.4 \\
\hline & & & & 0.6 & 96.3 & 1.0 \\
\hline & & & & 0.9 & 101.9 & 4.6 \\
\hline & \multirow{6}{*}{ Chicken } & \multirow{6}{*}{0.02} & \multirow{6}{*}{0.06} & 0.06 & 80.9 & 2.5 \\
\hline & & & & 0.075 & 88.8 & 5.2 \\
\hline & & & & 0.15 & 101.2 & 8.0 \\
\hline & & & & 0.3 & 105.7 & 2.5 \\
\hline & & & & 0.6 & 102.5 & 6.2 \\
\hline & & & & 0.9 & 98.4 & 10.4 \\
\hline
\end{tabular}

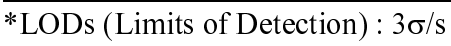

**LOQs (Limits of Quantitation) : 10 $/ \mathrm{s}$

$* * *$ Accuracy $($ Recovery \%) $=($ measure value/spiked value $) \times 100$ 


\section{Conclusion}

The analytical method devised to detectdicyclanil in chicken and lamb muscle using HPLC/UV-Vis and LC/MS/MS was found to be sensitive, reliable, and reproducible.

In chicken and lamb muscle, the LOD and LOQ were $0.02 \mathrm{mg} \mathrm{kg}^{-1}$ and $0.05 \sim 0.06 \mathrm{mg} \mathrm{kg}^{-1}$, respectively, and the intraday precision and accuracy of spiked samples were 2.3 10.4 RSD\% and 80.9 105.7\%, respectively. The described method satisfies the maximum residue limits (MRLs) of the Korean Food Code and has been used to analyze dicyclanil in chicken and lamb muscle by the Korean government and private laboratories.

\section{Acknowledgement}

This study was conducted with financial support from the National Institute of Food and Drug Safety Evaluation (NIFDS) of Korea

\section{References}

1. Stolker, A. A. M.; Brinkman, U. A. Th. J. Chromatogr. A 2005, 1067, 15.

2. Aerts, M. M. L.; Hogenboom, A. C.; Brinkman, U. A. Th. J. Chromatogr. B 1995, 667, 1.

3. The European Agency for the Evaluation of Medicinal Products, EMEA/MRL/733/00-Final, Committee for veterinary medicinal products, summary report (2), March 2000.

4. Sotiraki, S.; Stefanakis, A.; Hall, M. J. R.; Farkas, R.; Graf, J. F. Vet. Parasitol. 2005, 131, 107.

5. Yamada, R.; Kozono, M.; Ohmori, T.; Morimatsu, F.; Kityama, M. Biosci. Biotechnol. Biochem. 2006, 70, 54.

6. Department of Food Safety Ministry of Health, Labour and Welfare, Japan, Analytical Methods for Residual Compositional Substances of Agricultural Chemicals, Feed Additives, and Veterinary Drugs in Food, 2006.

7. Monographs prepared by the sixtieth meeting of the Joint FAO/WHO Expert Committee on Food Additives, Residues of some veterinary drugs in animals and foods, 2003, p31. 